\title{
Mus-Sasaki Metric and Harmonicity
}

\author{
Fethi Latti*, Mustapha Djaa and Abderrahim Zagane \\ (Communicated by Ákos G. HORVÁTH)
}

\begin{abstract}
In this paper, we introduce the Mus-Sasaki metric on the tangent bundle $T M$, as a new natural metric on $T M$. We establish necessary and sufficient conditions under which a vector field is harmonic with respect to the Mus-Sasaki metric. We also construct some examples of harmonic vector fields.
\end{abstract}

Keywords: Horizontal lift; vertical lift; Mus-Sasaki metric; harmonic vector field.

AMS Subject Classification (2010): Primary: 53A45 ; 53C20; Secondary: 58E20.

${ }^{*}$ Corresponding Author

\section{Introduction}

Consider a smooth map $\phi:\left(M^{m}, g\right) \rightarrow\left(N^{n}, h\right)$ between two Riemannian manifolds, then the energy functional is defined by

$$
E(\phi)=\frac{1}{2} \int_{M}|d \phi|^{2} d v_{g}
$$

(or over any compact subset $K \subset M$ ).

A map is called harmonic if it is a critical point of the energy functional $E$ (or $E(K)$ for all compact subsets $K \subset M)$. For any smooth variation $\{\phi\}_{t \in I}$ of $\phi$ with $\phi_{0}=\phi$ and $V=\left.\frac{d \phi_{t}}{d t}\right|_{t=0}$, we have

$$
\left.\frac{d}{d t} E\left(\phi_{t}\right)\right|_{t=0}=-\int_{M} h(\tau(\phi), V) d v_{g},
$$

where

$$
\tau(\phi)=\text { trace }_{g} \nabla d \phi
$$

is the tension field of $\phi$. Then $\phi$ is harmonic if and only if $\tau(\phi)=0$.

One can refer to [8], [9] , [12] for background on harmonic maps, and [4], [7] for background on generalized harmonic maps.

The existence and explicit construction of harmonic mappings between two given Riemannian manifolds (M, g) and $(\mathrm{N}, \mathrm{h})$ are two of the most fundamental problems of the theory of harmonic mappings. If $\mathrm{M}$ is compact and $\mathrm{N}$ has nonpositive sectional curvature, then any smooth map from $\mathrm{M}$ to $\mathrm{N}$ can be deformed into a harmonic map using the heat flow method [Eells and Sampson 1964]. However, there is no general existence theory of harmonic mappings if the target manifold does not satisfy the nonpositivity curvature condition. This fact makes it interesting to find harmonic maps defined by vector fields as a map from Riemannian manifold $(M, g)$ to its tangent bundle

Received : 17-05-2017, Accepted : 02-01-2018 
$T M$. Problems of this kind have been studied when TM is endowed with the Riemannian Sasaki metric see ([2] [10] [11] [12]) and with the Riemannian Cheeger-Gromoll metric (see [1]).

The main idea in this note consists in the modification of the Sasaki metric. First we introduce a new metric called Mus-Sasaki metric on the tangent bundle $T M$. This new natural metric will lead us to interesting results [18]. Afterward we establish necessary and sufficient conditions under which a vector field is harmonic with respect to the Mus-Sasaki metric (Theorem 3.2 and Theorem 3.3). We also construct some examples of harmonic vector fields and we give a formula for the construction of non trivial examples of vector fields (Theorem 3.5).

\section{Basic Notions and Definition on $T M$.}

\subsection{Horizontal and vertical lifts on $T M$.}

Let $(M, g)$ be an m-dimensional Riemannian manifold and $(T M, \pi, M)$ be its tangent bundle. A local chart $\left(U, x^{i}\right)_{i=1 \ldots n}$ on $M$ induces a local chart $\left(\pi^{-1}(U), x^{i}, y^{i}\right)_{i=1 \ldots n}$ on $T M$. Denote by $\Gamma_{i j}^{k}$ the Christoffel symbols of $g$ and by $\nabla$ the Levi-Civita connection of $g$.

We have two complementary distributions on $T M$, the vertical distribution $\mathcal{V}$ and the horizontal distribution $\mathcal{H}$, defined by :

$$
\begin{aligned}
\mathcal{V}_{(x, u)} & =\operatorname{Ker}\left(d \pi_{(x, u)}\right)=\left\{\left.a^{i} \frac{\partial}{\partial y^{i}}\right|_{(x, u)} ; \quad a^{i} \in \mathbb{R}\right\} \\
\mathcal{H}_{(x, u)} & =\left\{\left.a^{i} \frac{\partial}{\partial x^{i}}\right|_{(x, u)}-\left.a^{i} u^{j} \Gamma_{i j}^{k} \frac{\partial}{\partial y^{k}}\right|_{(x, u)} ; \quad a^{i} \in \mathbb{R}\right\},
\end{aligned}
$$

where $(x, u) \in T M$, such that $T_{(x, u)} T M=\mathcal{H}_{(x, u)} \oplus \mathcal{V}_{(x, u)}$.

Let $X=X^{i} \frac{\partial}{\partial x^{i}}$ be a local vector field on $M$. The vertical and the horizontal lifts of $X$ are defined by

$$
\begin{aligned}
X^{V} & =X^{i} \frac{\partial}{\partial y^{i}} \\
X^{H} & =X^{i} \frac{\delta}{\delta x^{i}}=X^{i}\left\{\frac{\partial}{\partial x^{i}}-y^{j} \Gamma_{i j}^{k} \frac{\partial}{\partial y^{k}}\right\}
\end{aligned}
$$
$T T M$.

For consequences, we have $\left(\frac{\partial}{\partial x^{i}}\right)^{H}=\frac{\delta}{\delta x^{i}}$ and $\left(\frac{\partial}{\partial x^{i}}\right)^{V}=\frac{\partial}{\partial y^{i}}$, then $\left(\frac{\delta}{\delta x^{i}}, \frac{\partial}{\partial y^{i}}\right)_{i=1 \ldots n}$ is a local adapted frame in Remark 2.1. .

1. if $w=w^{i} \frac{\partial}{\partial x^{i}}+\bar{w}^{j} \frac{\partial}{\partial y^{j}} \in T_{(x, u)} T M$, then its horizontal and vertical parts are defined by

$$
\begin{aligned}
w^{h} & =w^{i} \frac{\partial}{\partial x^{i}}-w^{i} u^{j} \Gamma_{i j}^{k} \frac{\partial}{\partial y^{k}} \in \mathcal{H}_{(x, u)} \\
w^{v} & =\left\{\bar{w}^{k}+w^{i} u^{j} \Gamma_{i j}^{k}\right\} \frac{\partial}{\partial y^{k}} \in \mathcal{V}_{(x, u)}
\end{aligned}
$$

2. if $u=u^{i} \frac{\partial}{\partial x^{i}} \in T_{x} M$ then its vertical and horizontal lifts are defined by

$$
\begin{aligned}
u^{V} & =u^{i} \frac{\partial}{\partial y^{i}} \in \mathcal{V}_{(x, u)} \in \mathcal{H}_{(x, u)} \\
u^{H} & =u^{i}\left\{\frac{\partial}{\partial x^{i}}-y^{j} \Gamma_{i j}^{k} \frac{\partial}{\partial y^{k}}\right\} .
\end{aligned}
$$


Proposition 2.1 ([17]). Let $(M, g)$ be a Riemannian manifold and $R$ its tensor curvature, then for all vector fields $X, Y \in$ $\Gamma(T M)$ and $p \in T^{2} M$ we have:

1. $\left[X^{H}, Y^{H}\right]_{p}=[X, Y]_{p}^{H}-\left(R_{x}(X, Y) u\right)^{V}$,

2. $\left[X^{H}, Y^{V}\right]_{p}=\left(\nabla_{X} Y\right)_{p}^{V}$,

3. $\left[X^{V}, Y^{V}\right]_{p}=0$,

where $p=(x, u)$.

\section{Mus-Sasaki metric.}

3.1 Mus-Sasaki metric.

Definition 3.1 ([18]). Let $(M, g)$ be a Riemannian manifold and $f: M \times \mathbb{R} \rightarrow] 0,+\infty[$. On the tangent bundle $T M$, we define a Mus-Saski metric noted $g_{f}^{S}$ by

1. $g_{f}^{S}\left(X^{H}, Y^{H}\right)_{(x, u)}=g_{x}(X, Y)$

2. $g_{f}^{S}\left(X^{H}, Y^{V}\right)_{(x, u)}=0$

3. $g_{f}^{S}\left(X^{V}, Y^{V}\right)_{(x, u)}=f(x, r) g_{x}(X, Y)$

where $X, Y \in \Gamma(T M),(x, u) \in T M$ and $r=g(u, u) . f$ is called twisting function.

Note that, if $f=1$ then $g_{f}^{S}$ is the Sasaki metric [17].

The notion of Sasaki metric and Gromol-Chegeer was considered in [3], [13], [14], [15], [16], [17].

Lemma 3.1 ([18]). Let $(M, g)$ be a Riemannian manifold, then for all $x \in M$ and $u=u^{i} \frac{\partial}{\partial x^{i}} \in T_{x} M$, we have the following

1. $X^{H}(g(u, u))_{(x, u)}=0$

2. $X^{H}(g(Y, u))_{(x, u)}=g\left(\nabla_{X} Y, u\right)_{x}$

3. $X^{V}\left(g(u, u)_{(x, u)}=2 g(X, u)_{x}\right.$

4. $X^{V}\left(g(Y, u)_{(x, u)}=g(X, Y)_{x}\right.$

From Lemma 3.2, we obtain

Lemma 3.2. Let $(M, g)$ be a Riemannian manifold, $\left.F:(s, t) \in \mathbb{R}^{2} \rightarrow F(s, t) \in\right] 0,+\infty[, \alpha: M \rightarrow] 0,+\infty[$ and $\beta: \mathbb{R} \rightarrow$ ] $0,+\infty[$ be smooth functions. If $f(x, r)=F(\alpha(x), \beta(r))$, then we have the following

1. $X^{V}(f)_{(x, u)}=2 \beta^{\prime}(r) g_{x}(X, u) \frac{\partial F}{\partial t}(\alpha(x), \beta(r))$

2. $X^{H}(f)_{(x, u)}=g_{x}\left(\operatorname{grad}_{M} \alpha, X\right) \frac{\partial F}{\partial s}(\alpha(x), \beta(r))$

where $(x, u) \in T M$ and $r=g_{x}(u, u)$.

In the following, we consider $f(x, r)=F(\alpha(x), \beta(r))$, where $\left.F:(s, t) \in \mathbb{R}^{2} \rightarrow F(s, t) \in\right] 0,+\infty[, \alpha: M \rightarrow] 0,+\infty[$ and $\beta: \mathbb{R} \rightarrow] 0,+\infty[$ are smooth functions. 
Theorem 3.1 ([18]). Let $(M, g)$ be a Riemannian manifold. If $f(x, r)=F(\alpha(x), \beta(r))$ and $\nabla$ (resp $\widetilde{\nabla})$ denote the Levi-Civita connection of $(M, g)$ (resp $\left(T M, g_{f}^{S}\right)$, then we have:

$$
\begin{aligned}
\text { 1. }\left(\widetilde{\nabla}_{X^{H}} Y^{H}\right)_{p}= & \left(\nabla_{X} Y\right)_{p}^{H}-\frac{1}{2}\left(R_{x}(X, Y) u\right)^{V}, \\
\text { 2. }\left(\widetilde{\nabla}_{X^{H}} Y^{V}\right)_{p}= & \left(\nabla_{X} Y\right)_{p}^{V}+\frac{f(x, r)}{2}\left(R_{x}(u, Y) X\right)^{H} \\
& +\frac{1}{2 f(x, r)} g_{x}\left(\operatorname{grad}_{M} \alpha, X\right) \frac{\partial F}{\partial s}(\alpha(x), \beta(r)) Y_{p}^{V} \\
\text { 3. }\left(\widetilde{\nabla}_{X^{V}} Y^{H}\right)_{p}= & \left.\frac{f(x, r)}{2}\left(R_{x}(u, X) Y\right)\right)^{H}+\frac{1}{2 f(x, r)} g_{x}\left(\operatorname{grad}_{M} \alpha, Y\right) \frac{\partial F}{\partial s}(\alpha(x), \beta(r)) X_{p}^{V} \\
\text { 4. }\left(\widetilde{\nabla}_{X^{V}} Y^{V}\right)_{p}= & \left.\left.\frac{\beta^{\prime}(r)}{f(x, r)} \frac{\partial F}{\partial t}(\alpha(x), \beta(r))\left[g_{x}(Y, U) X_{p}^{V}\right)+g_{x}(X, U) Y_{p}^{V}\right)-g_{x}(X, Y) U_{p}^{V}\right] \\
& -\frac{1}{2} g_{x}(X, Y) \frac{\partial F}{\partial t}(\alpha(x), \beta(r))\left(\operatorname{grad}_{M} \alpha\right)_{p}^{H} .
\end{aligned}
$$

for all vector fields $X, Y \in \Gamma(T M)$ and $p=(x, u) \in T M$, where $R$ denote the curvature tensor of $(M, g)$.

The proof of Theorem 3.1 follows directly from Kozul formula, Lemma 3.1 and Lemma 3.2.

Lemma 3.3. Let $(M, g)$ be a Riemannian manifold. If $X, Y \in \Gamma(T M)$ are a vector fields and $(x, u) \in T M$ such that $X_{x}=u$, then we have

$$
d_{x} X\left(Y_{x}\right)=Y_{(x, u)}^{H}+\left(\nabla_{Y} X\right)_{(x, u)}^{V} .
$$

Proof. Let $\left(U, x^{i}\right)$ be a local chart on $M$ in $x \in M$ and $\left(\pi^{-1}(U), x^{i}, y^{j}\right)$ be the induced chart on $T M$, if $X_{x}=$ $\left.X^{i}(x) \frac{\partial}{\partial x^{i}}\right|_{x}$ and $Y_{x}=\left.Y^{i}(x) \frac{\partial}{\partial x^{i}}\right|_{x}$, then

$$
d_{x} X\left(Y_{x}\right)=\left.Y^{i}(x) \frac{\partial}{\partial x^{i}}\right|_{\left(x, X_{x}\right)}+\left.Y^{i}(x) \frac{\partial X^{k}}{\partial x^{i}}(x) \frac{\partial}{\partial y^{k}}\right|_{\left(x, X_{x}\right)},
$$

thus the horizontal part is given by

$$
\begin{aligned}
\left(d_{x} X\left(Y_{x}\right)\right)^{h} & =\left.Y^{i}(x) \frac{\partial}{\partial x^{i}}\right|_{\left(x, X_{x}\right)}-\left.Y^{i}(x) X^{j}(x) \Gamma_{i j}^{k}(x) \frac{\partial}{\partial y^{k}}\right|_{\left(x, X_{x}\right)} \\
& =Y_{\left(x, X_{x}\right)}^{H}
\end{aligned}
$$

and the vertical part is given by

$$
\begin{aligned}
\left(d_{x} X\left(Y_{x}\right)\right)^{v} & =\left.\left\{Y^{i}(x) \frac{\partial X^{k}}{\partial x^{i}}(x)+Y^{i}(x) X^{j}(x) \Gamma_{i j}^{k}(x)\right\} \frac{\partial}{\partial y^{k}}\right|_{\left(x, X_{x}\right)} \\
& =\left(\nabla_{Y} X\right)_{\left(x, X_{x}\right)}^{V} .
\end{aligned}
$$

Lemma 3.4. Let $(M, g)$ be a Riemannian n-dimensional manifold and $\left(T M, g_{f}^{S}\right)$ be its tangent bundle equipped with the Mus-Sasaki metric . if $X \in \Gamma(T(T M))$, then the energy density associated to $X$ is given by

$$
e(X)=\frac{n}{2}+\frac{f}{2} \operatorname{trace}_{g} g(\nabla X, \nabla X) .
$$

Proof. Let $\left(e_{1}, \ldots, e_{n}\right)$ be a local orthonormal frame on $M$, then

$$
2 e(X)_{p}=\sum_{i=1}^{n} g_{f}^{S}\left(d X\left(e_{i}\right), d X\left(e_{i}\right)\right)
$$

Using Lemma 3.3 , we obtain 


$$
\begin{aligned}
2 e(X) & \left.=\sum_{i=1}^{n} g_{f}^{S}\left(e_{i}^{H}, e_{i}^{H}\right)+\sum_{i=1}^{n} g_{f}^{S}\left(\nabla_{e_{i}} X\right)^{V},\left(\nabla_{e_{i}} X\right)^{V}\right) \\
& =\sum_{i=1}^{n} g\left(e_{i}, e_{i}\right)+\sum_{i=1}^{n} f g\left(\nabla_{e_{i}} X, \nabla_{e_{i}} X\right) \\
& =n+\text { frace }_{g} g(\nabla X, \nabla X) .
\end{aligned}
$$

Theorem 3.2. Let $(M, g)$ be a Riemannian manifold and $\left(T M, g_{f}^{S}\right)$ be its tangent bundle equipped with the twisted Sasaki metric, such as $f(x, r)=F(\alpha(x), \beta(r))$. Then the tension field associated with $X \in \Gamma(T(T M))$ is given by:

$$
\begin{aligned}
\tau(X)=( & \left.\frac{1}{f(x, r)} \frac{\partial F}{\partial s}(\alpha(x), \beta(r)) \nabla_{\operatorname{grad}(\alpha)} X\right)^{V}+\left(\text { trace }_{g} A(X)\right)^{V} \\
& +\left(\text { trace }_{g} B(X)\right)^{H} .
\end{aligned}
$$

where $A(X)$ and $B(X)$ are a bilinear maps defined by:

$$
\begin{aligned}
& A(X)=\nabla^{2} X+\frac{\beta^{\prime}(r)}{f(x, r)} \frac{\partial F}{\partial t}(\alpha(x), \beta(r))[2 g(\nabla X, X) \nabla X-g(\nabla X, \nabla X) X] \\
& B(X)=f(x, r) R(X, \nabla X) *-\frac{1}{2} g(\nabla X, \nabla X) \frac{\partial F}{\partial t}(\alpha(x), \beta(r)) \operatorname{grad}_{M}(\alpha) .
\end{aligned}
$$

Proof. Let $x \in M$ and $\left\{e_{i}\right\}_{i=1}^{n}$ be a local orthonormal frame on $M$ such that $\nabla_{e_{i}} e_{j}=0$ at $x$ and $X_{x}=u$, then by summing over $i$, we have

$$
\begin{aligned}
\tau(X)_{x} & =\left[\widetilde{\nabla}_{d X\left(e_{i}\right)} d X\left(e_{i}\right)\right] \\
& =\left[\widetilde{\nabla}_{e_{i}^{H}+\left(\nabla_{e_{i}} X\right)^{V}}\left(e_{i}^{H}+\left(\nabla_{e_{i}} X\right)^{V}\right)\right] \\
& =\left[\widetilde{\nabla}_{e_{i}^{H}} e_{i}^{H}+\widetilde{\nabla}_{e_{i}^{H}}\left(\nabla_{e_{i}} X\right)^{V}+\widetilde{\nabla}_{\left(\nabla_{e_{i}} X\right)^{V}} e_{i}^{H}+\widetilde{\nabla}_{\left(\nabla_{e_{i}} X_{\sigma}\right)^{V}}\left(\nabla_{e_{i}} X_{\sigma}\right)^{V}\right]
\end{aligned}
$$

by Theorem 3.1 we obtain

$$
\begin{aligned}
\widetilde{\nabla}_{e_{i}^{H}} e_{i}^{H}= & 0 \\
\widetilde{\nabla}_{e_{i}^{H}}\left(\nabla_{e_{i}} X\right)^{V}= & \frac{f(x, r)}{2}\left(R_{x}\left(X_{x}, \nabla_{e_{i}} X\right) e_{i}\right)^{H}+\frac{1}{2 f(x, r)} \frac{\partial F}{\partial s}(\alpha(x), \beta(r))\left(\nabla_{\operatorname{grad}_{M}} X\right)_{p}^{V} \\
& +\left(\nabla_{e_{i}} \nabla_{e_{i}} X\right)^{V} \\
\widetilde{\nabla}_{\left(\nabla_{e_{i}} X\right)^{V} e_{i}^{H}}= & \left.\frac{f(x, r)}{2}\left(R_{x}\left(X_{x}, \nabla_{e_{i}} X\right) e_{i}\right)\right)^{H}+\frac{1}{2 f(x, r)} \frac{\partial F}{\partial s}(\alpha(x), \beta(r))\left(\nabla_{g_{r a d} \alpha} X\right)_{p}^{V} \\
\widetilde{\nabla}_{\left(\nabla_{e_{i}} X_{\sigma}\right)^{V}}\left(\nabla_{e_{i}} X_{\sigma}\right)^{V}= & -\frac{1}{2} g_{x}\left(\nabla_{e_{i}} X, \nabla_{e_{i}} X\right) \frac{\partial F}{\partial t}(\alpha(x), \beta(r))\left(\operatorname{grad}_{M} \alpha\right)_{p}^{H} \\
& +\frac{\beta^{\prime}(r)}{f(x, r)} \frac{\partial F}{\partial t}(\alpha(x), \beta(r))\left[2 g_{x}\left(\nabla_{e_{i}} X, X_{x}\right)\left(\nabla_{e_{i}} X\right)_{p}^{V}\right) \\
& \left.-g_{x}\left(\nabla_{e_{i}} X, \nabla_{e_{i}} X\right) X_{x}^{V}\right] .
\end{aligned}
$$

From Theorem 3.2 we obtain

Theorem 3.3. Let $(M, g)$ be a Riemannian manifold and $\left(T M, g_{f}^{S}\right)$ be its tangent bundle equipped with the Mus-Sasaki metric, such as $f(x, r)=F(\alpha(x), \beta(r))$. A vector field $X \in \Gamma(T M)$ is harmonic if and only the following conditions are 
verified

$$
\begin{aligned}
0=\operatorname{trace}_{g}\left\{\nabla^{2} X+\right. & \left.\frac{\beta^{\prime}(r)}{f(x, r)} \frac{\partial F}{\partial t}(\alpha(x), \beta(r))\left[2 g_{x}(\nabla X, X) \nabla X-g_{x}(\nabla X, \nabla X) X\right]\right\} \\
& +\frac{1}{f(x, r)} \frac{\partial F}{\partial s}(\alpha(x), \beta(r)) \nabla_{\operatorname{grad}(\alpha)} X
\end{aligned}
$$

and

$0=f(x, r) \operatorname{trace}_{g} R(X, \nabla X) *-\frac{1}{2} \frac{\partial F}{\partial t}(\alpha(x), \beta(r)) \operatorname{trace}_{g} g_{x}(\nabla X, \nabla X) \operatorname{grad}_{M}(\alpha)$.

where $r=g\left(X_{x}, X_{x}\right)=\left\|X_{x}\right\|^{2}$.

Corollary 3.1. Let $(M, g)$ be a Riemannian manifold and $\left(T M, g_{f}^{S}\right)$ be its tangent bundle equipped with the Mus-Sasaki metric. If $X \in \Gamma(T M)$ is a parallel vector field (i.e $\nabla X=0$ ), then $X$ is harmonic.

Theorem 3.4. Let $(M, g)$ be a Riemannian compact manifold and $\left(T M, g_{f}^{S}\right)$ be its tangent bundle equipped with the MusSasaki metric. Then $X \in \Gamma(T M)$ is a harmonic vector field if and only if $X$ is parallel.

Proof. . If $X$ is parallel, from Corollary 3.1, we deduce that $X$ is harmonic vector field. Inversely:

Let $X_{t}$ be a compactly supported variation of $X$ defined by $X_{t}=(1+t) X$. From Lemma 3.4 we have

$$
e\left(X_{t}\right)=\frac{n}{2}+f(x, r) \frac{(t+1)^{2}}{2} \operatorname{trace}_{g} g(\nabla X, \nabla X)
$$

If $X$ is a critical point of the energy functional, then we have :

$$
\begin{aligned}
0 & =\frac{d}{d t} E\left(X_{t}\right)_{\mid t=0} \\
& =\frac{d}{d t}\left[\int_{M}\left(\frac{n}{2}+f(x, r) \frac{(t+1)^{2}}{2} \text { trace }_{g} g(\nabla X, \nabla X)\right) d v_{g}\right]_{t=0} \\
& =\int_{M} f(x, r) \text { trace }_{g} g(\nabla X, \nabla X) d v_{g}
\end{aligned}
$$

then

$$
g(\nabla X, \nabla X)=0
$$

Example 3.1. Let $S^{1}=\left\{(x, y) \in \mathbb{R}^{2}\right\}$ endowed with the natural metric $d x^{2}+d y^{2}$ and $X:(x, y) \in S^{1} \rightarrow$ $(x, y,-y, x) \in T^{2} S^{1}$, we have: $\nabla^{S^{1}} X=0$. From Corollary 3.1 we deduce that $X$ is harmonic section.

Example 3.2. If $S^{2} \times \mathbb{R}$ is endowed with the product of canonical metric, then the vector field $X=\frac{\partial}{\partial t}$ is harmonic.

Example 3.3. If $\mathbb{R}^{n}$ is endowed with the canonical metric, then any constant vector field $X$ on $\mathbb{R}^{n}$ is harmonic.

Remark 3.1. In general, using Corollary 3.1 and Theorem 3.4 we can construct many examples for harmonic vector fields.

From Theorem 3.3 we deduce

Theorem 3.5. Let $\left(\mathbb{R}^{n},<>\right)$ the real euclidian space and $T \mathbb{R}^{n}$ its tangent bundle equipped with the Mus-Sasaki metric, such as $f(x, r)=F(\alpha(x), \beta(r))$. If $X=\left(X^{1}, \ldots, X^{n}\right)$ is a vector field on $\mathbb{R}^{n}$, then $X$ is harmonic if and only if

$$
\begin{aligned}
0= & \left.\sum_{i}\left[\frac{1}{\left.f\left(x,\|X\|^{2}\right)\right)} \frac{\partial \alpha}{\partial x^{i}} \frac{\partial X^{k}}{\partial x^{i}} \frac{\partial F}{\partial s}\left(\alpha(x), \beta\left(\|X\|^{2}\right)\right)\right)+\frac{\partial^{2} X^{k}}{\partial x_{i}^{2}}\right] \\
& \left.+\frac{\beta^{\prime}\left(\|X\|^{2}\right)}{f\left(x,\|X\|^{2}\right)} \frac{\partial F}{\partial t}\left(\alpha(x), \beta\left(\|X\|^{2}\right)\right)\right) \sum_{i j}\left[2 X^{j} \frac{\partial X^{j}}{\partial x^{i}} \frac{\partial X^{k}}{\partial x^{i}}-X^{k}\left(\frac{\partial X^{j}}{\partial x_{i}}\right)^{2}\right] \\
0= & \frac{\partial \alpha}{\partial x^{k}}(x) \frac{\partial F}{\partial t}\left(\alpha(x), \beta\left(\|X\|^{2}\right)\right) \sum_{i j}\left(\frac{\partial X^{j}}{\partial x_{i}}\right)^{2} .
\end{aligned}
$$


for all $k=1, \ldots, n$.

Corollary 3.2. Let $\left(\mathbb{R}^{n},<>\right)$ the real euclidian space and $T \mathbb{R}^{n}$ its tangent bundle equipped with the Mus-Sasaki metric. If $\alpha$ and $\beta$ are constant functions, or $F$ is a constant function, then $X=\left(X^{1}, \ldots, X^{n}\right)$ is a harmonic vector field on $\mathbb{R}^{n}$ if and only if for all $i=1, \ldots, n ., X^{i}$ is a real harmonic function on $\mathbb{R}^{n}$.

Corollary 3.3. Let $\left(\mathbb{R}^{n},<>\right)$ the real euclidian space and $T \mathbb{R}^{n}$ its tangent bundle equipped with the Mus-Sasaki metric. If $(\operatorname{grad} \alpha \neq 0)$ and $\left(\frac{\partial F}{\partial t} \neq 0\right)$ then $X$ is a harmonic vector field on $\mathbb{R}^{n}$ if and only if $X$ is constant.

Corollary 3.4. Let $\left(\mathbb{R}^{n},<>\right)$ the real euclidian space and $T \mathbb{R}^{n}$ its tangent bundle equipped with the Mus-Sasaki metric. If $\alpha$ is a constant function then $X=\left(X^{1}, \ldots, X^{n}\right)$ is a harmonic vector field on $\mathbb{R}^{n}$ if and only if $X$ verifies the following system of equations

$$
\left.\sum_{i} \frac{\partial^{2} X^{k}}{\partial x_{i}^{2}}+\frac{\beta^{\prime}\left(\|X\|^{2}\right)}{f\left(x,\|X\|^{2}\right)} \frac{\partial F}{\partial t}\left(\alpha(x), \beta\left(\|X\|^{2}\right)\right)\right) \sum_{i j}\left[2 X^{j} \frac{\partial X^{j}}{\partial x^{i}} \frac{\partial X^{k}}{\partial x^{i}}-X^{k}\left(\frac{\partial X^{j}}{\partial x_{i}}\right)^{2}\right]=0
$$

for all $k=1, \ldots, n$.

Remark 3.2. Using Corollary 3.4, we can construct many examples of nontrivial harmonic vector fields.

Example 3.4. If $\mathbb{R}^{n}$ is endowed with the canonical metric and $T \mathbb{R}^{n}$ its tangent bundle equipped with the Mus-Sasaki metric, such as $F(s, t)=t(i . e: f(x, r)=\beta(r))$. From Corollary 3.4, we deduce that $X=\left(h\left(x_{1}\right), 0, \ldots ., 0\right)$ is a harmonic vector field if and only the function $h$ is solution of differential equation:

$$
h^{\prime \prime}+\frac{\beta^{\prime}\left(h^{2}\right)}{\beta\left(h^{2}\right)} h\left(h^{\prime}\right)^{2}=0
$$

or

$$
h^{\prime \prime}+\frac{\left(\beta\left(h^{2}\right)\right)^{\prime}}{2 \beta\left(h^{2}\right)} h^{\prime}=0
$$

when $\beta(t)=t$, the solution of differential equation (3.3) is given by $h\left(x_{1}\right)=\sqrt{a x_{1}+b}$. when $\beta(t)=\frac{1}{t}$, the solution of differential equation (3.3) is given by $h\left(x_{1}\right)=b \exp \left(a x_{1}\right)$.

\section{Acknowledgement:}

The authors are supported by Saida Laboratory of Geometry Analysis Controls and Applications and Algerian research agency CNEPRU.

\section{References}

[1] Boeckx E. and Vanhecke L., Harmonic and minimal vector fields on tangent and unit tangent bundles, Differential Geometry and its Applications Volume 13, Issue 1, July 2000, Pages 77-93.

[2] Calvaruso G., Naturally Harmonic Vector Fields, Note di Matematica, Note Mat. 1(2008), suppl. n. 1, 107-130

[3] Cengiz, N., Salimov, A.A.: Diagonal lift in the tensor bundle and its applications. Appl. Math. Comput. 142, no. 2-3, 309-319 (2003).

[4] Djaa M., Mohamed Cherif A., Zegga K. And Ouakkas S., On the Generalized of Harmonic and Bi-harmonic Maps, international electronic journal ofgeometry, 5 no. 1(2012), 90-100.

[5] Djaa M., Gancarzewicz J., The geometry of tangent bundles of order r, Boletin Academia , Galega de Ciencias ,Espagne, 4 (1985), 147-165

[6] Djaa N.E.H., Ouakkas S. , M. Djaa, Harmonic sections on the tangent bundle of order two. Annales Mathematicae et Informaticae 38( 2011) pp 15-25. 1.

[7] Djaa N.E.H., Boulal A. and Zagane A., Generalized warped product manifolds and Biharmonic maps, Acta Math. Univ. Comenianae Vol. LXXXI, 2 (2012), pp. 283-298

[8] Eells J., Sampson J.H., Harmonic mappings of Riemannian manifolds. Amer. J. Maths. 86(1964). 
[9] Eells, J. and Lemaire, L., Another report on harmonic maps, Bull. London Math. Soc. 20(1988), 385-524.

[10] Ishihara T., Harmonic sections of tangent bundles. J. Math. Tokushima Univ. 13 (1979), 23-27.

[11] Konderak J.J., On Harmonic Vector Fields, Publications Matematiques. Vol 36 (1992), 217-288.

[12] Opriou V., On Harmonic Maps Between Tangent Bundles. Rend. Sem. Mat, Vol 47, 1 (1989).

[13] Salimov, A., Gezer, A., Akbulut, K.: Geodesics of Sasakian metrics on tensor bundles. Mediterr. J. Math. 6, no.2, 135-147 (2009).

[14] Salimov, A., Gezer, A., On the geometry of the (1, 1)-tensor bundle with Sasaki type metric. Chinese Annals of Mathematics, Series B May 2011, Volume 32, Issue 3, pp 369-386.

[15] Salimov A. and Agca F. ,Some Properties of Sasakian Metrics in Cotangent Bundles. Mediterranean Journal of Mathematics; 8(2) (2011). 243-255.

[16] Salimov A. A. and Kazimova S., Geodesics of the Cheeger-Gromoll Metric, Turk J Math 33 (2009) , 99 - 105.

[17] Yano K., Ishihara S. Tangent and Cotangent Bundles, Marcel Dekker. INC. New York 1973.

[18] A. Zagane and M. Djaa, On geodesics of warped Sasaki metric, Mathematical sciences and Applications E-Notes. Vol1 (2017), 85-92.

\section{Affiliations}

F. LATTI

AdDress: Saida University, Dept. of mathematics, 20000, Saida-Algeria.

E-MAIL: Lattifethi2017@gmail.com

ORCID ID: $0000-0002-5920-9699$

M. DJAA

AdDRESS: Relizane University, Dept. of mathematics, 48000, Relizane-Algeria. E-MAIL: Djaamustapha20@gmail.com

ORCID ID: $0000-0002-7330-2144$

A. ZAGANE

AdDress: Relizane University, Dept. of mathematics, 48000, Relizane-Algeria.

E-MAIL: Zagane2016@gmail.com

ORCID ID: $0000-0001-9339-3787$ 